\title{
Sensorlose Regelung einer Synchron-Reluktanzmaschine im gesamten Drehzahl- und Lastbereich einschließlich Stillstand
}

\author{
M. Hofer, M. Izaak, M. Schrödl
}

\begin{abstract}
In dieser Arbeit wird die sensorlose Regelung einer Synchron-Reluktanzmaschine (SynRM) untersucht. Zur Ermittlung der Rotorposition bei kleinen Drehzahlen einschließlich Stillstand wird das sogenannte INFORM-Verfahren eingesetzt. Die dazu benötigte Stromanstiegsmessung wird in das Pulsmuster des Spannungszwischenkreisumrichters integriert, um eine zusätzliche Geräuschentwicklung verglichen mit bisherigen spannungsinjizierenden sensorlosen Verfahren zu vermeiden und gleichzeitig eine hohe Bandbreite der Positionsbestimmung zu erhalten. Bei höheren Drehzahlen wird ein EMK-Modell für SynRM vorgestellt und mittels Messungen verifiziert. Die damit dargestellte geberlose Regelung einer SynRM im gesamten Drehzahlbereich inklusive Stillstand stellt schließlich einen attraktiven und kostengünstigen elektrischen Antrieb für unterschiedliche Anwendungen dar.
\end{abstract}

Schlüsselwörter: Synchron-Reluktanzmaschine; sensorlose Regelung; elektrische Antriebe; INFORM-Verfahren; EMK-Modell

\begin{abstract}
Sensorless control of a synchronous reluctance machine in the whole speed-and loadrange including standstill.
In this paper a position sensorless control of a synchronous reluctance machine (SynRM) is discussed. The INFORM method is used to obtain the angular position information at low rotational speeds and standstill. By integration of current slope measurement into the pulse width pattern of the voltage source inverter a high position detection rate is reached at no additional accoustic noise compared to sensorless methods using voltage injection. At high rotational speeds an EMF model for SynRMs is presented and verified by experiments. Thus, a sensorless control of SynRMs in the complete speed range including standstill is implemented and offers an attractive and cost efficient electric drive solution for several applications.
\end{abstract}

Keywords: reluctance synchronous machine; sensorless control; electrical drive; INFORM method; EMF model

Eingegangen am 6. November 2015, angenommen am 18. Dezember 2015, online publiziert am 2. März 2016 (c) The Author(s) 2016. Dieser Artikel ist auf Springerlink.com mit Open Access verfügbar

\begin{abstract}
1. Einleitung
Der Einsatz von Synchron-Reluktanzmaschinen (SynRM) in der elektrischen Antriebstechnik hat in den letzten Jahren an Bedeutung gewonnen. Beispielsweise wird im Bereich der Elektromobilität aus Kostengründen eine Alternative zu PermanentmagneterregtenSynchronmaschinen (PSM) aufgrund des hohen Preises für SeltenErd-Magnete untersucht. Die Preise haben sich derzeit wieder normalisiert, sind jedoch nach wie vor ca. doppelt so hoch wie vor 4-5 Jahren. Als eine mögliche Alternative wird auch die SynRM in Betracht gezogen [1]. Die Drehmomentendichte ist zwar geringer als bei PSM Antrieben, aber da bei reinen Elektrofahrzeugen im Gegesatz zu Hybrid-Antrieben die kompakte Baugröße nicht die höchste Priorität besitzt, sind hier permanentmagnetlose elektrische Maschinen wie SynRM oder Asynchronmaschinen (ASM) denkbar. Auch in industriellen Anwendungen werden durch den Gesetzgeber für Elektromotoren bzw. elektrische Antriebe stetig höhere Wirkungsgrade gefordert. Zur Erfüllung der Wirkungsgradklasse IE4 (Super Premium Efficiecy) nach Norm IEC 60034-30 bieten manche Elektromotorenhersteller anstatt ASM nun SynRM an. Die Untersuchungen in [2] und [3] zum Einsatz von SynRM anstatt ASM hinsichtlich Baugröße und Effizienz zeigen, dass SynRM bei gleichem Volumen besseren Wirkungsgrad haben, oder bei gleichem Wirkungsgrad kleiner bauen, oder eben beide Eigenschaften als Kompromiss vereinen, nämlich sowohl eine leichte Verbesserung im Wirkungsgrad
\end{abstract}

als auch eine geringe Reduktion der Motorgröße möglich ist. SynRM haben im Vergleich zu ASM oder PSM einen sehr einfachen Aufbau welcher in Abb. 1 ersichtlich ist. Der Stator trägt wie eine ASM oder PSM eine Drehstromwicklung, der Rotor hingegen besteht nur aus dem Rotorblechpaket und hat weder Permanentmagnete, einen Kurzschlusskäfig oder eine Wicklung. Dabei erfolgt die Drehmomentbildung aufgrund der speziellen geometrischen Ausformung des Rotors und den damit verbundenen Reluktanzeffekten durch unterschiedliche magnetische Widerstände.

Der Betrieb der SynRM erfolgt mittels feldorientierter Regelung durch einen Frequenzumrichter. Dazu wird die Lagewinkelinformation des Rotors bezogen auf den Stator benötigt, welche üblicherweise durch einen Lagegeber bestimmt wird. Alternativ kann diese Winkelinformation durch geberlose Verfahren, wie das in dieser Arbeitet verwendete INFORM-Verfahren zur sogenannten sensorlosen Regelung von SynRM angewendet werden [4].

Hofer, Matthias, Institut für Energiesysteme und Elektrische Antriebe, Gußhausstraße 25, 1040 Wien, Österreich (E-Mail: matthias.hofer@tuwien.ac.at); Izaak, Martin, Institut für Energiesysteme und Elektrische Antriebe, Gußhausstraße 25, 1040 Wien, Österreich (E-Mail: martin.izaak@tuwien.ac.at); Schrödl, Manfred, Institut für Energiesysteme und Elektrische Antriebe, Gußhausstraße 25, 1040 Wien, Österreich

(E-Mail: manfred.schroedl@tuwien.ac.at) 


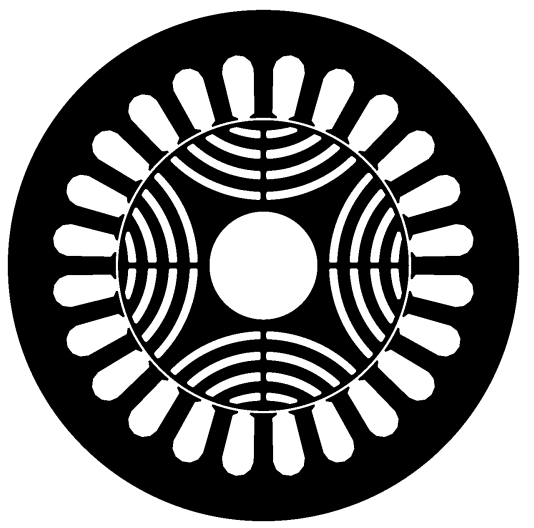

Abb. 1. Schnittbild einer 4-poligen Synchron-Reluktanzmaschine mit 4 Flussbarrieren

Tab. 1. Nenndaten der Synchron-Reluktanzmaschine

\begin{tabular}{ll}
\hline Nennleistung & $20 \mathrm{~kW}$ \\
Nennmoment & $65 \mathrm{~N} \mathrm{~m}$ \\
Nenndrehzahl & $2940 \mathrm{~min}^{-1}$ \\
Nennstrom & $370 \mathrm{~A}$ \\
Nennspannung & $\mathrm{Y} 3 \times 44 \mathrm{~V}$ \\
\hline
\end{tabular}

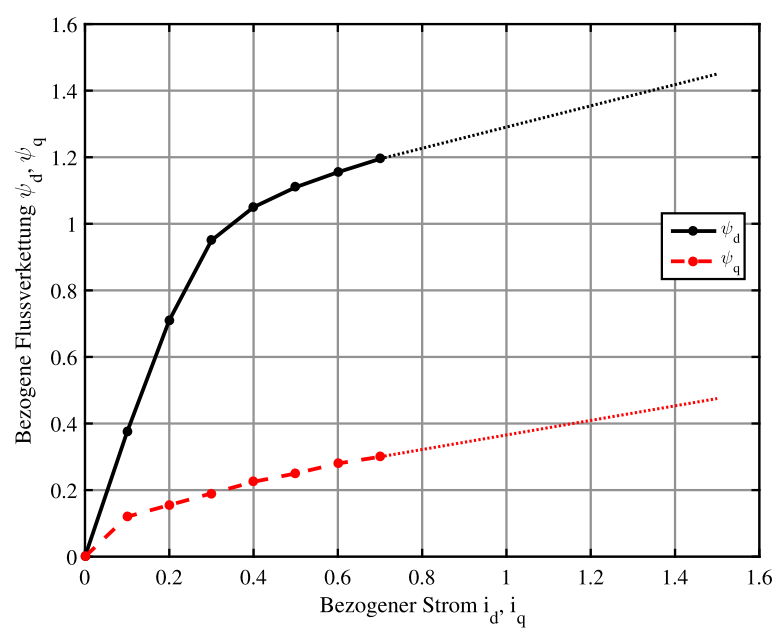

Abb. 2. Flussverkettungen $\psi_{d}$ und $\psi_{q}$ in Abhängigkeit des jeweiligen Stroms $i_{d}$ bzw. $i_{q}$

\section{Synchron-Reluktanzmaschine}

Die mathematische Beschreibung der SynRM erfolgt wie bei PSM oder elektrisch erregten Synchronmaschinen vorzugsweise im rotorfesten $(d, q)$-Koordinatensystem, wobei die $d$-Achse mit der magnetischen Vorzugsrichtung aufgrund des geringsten magnetischen Widerstandes des Rotors übereinstimmt. Die Flussverkettung des Stators lässt sich daher folgendermaßen angeben.

$$
\begin{aligned}
& \psi_{S, d}=I_{d}\left(i_{d}\right) \cdot i_{d} \\
& \psi_{S, q}=I_{q}\left(i_{q}\right) \cdot i_{q}
\end{aligned}
$$

Die gemessenen Flussverkettungen $\psi_{s, d}\left(i_{d}\right)$ in Längs- und $\psi_{s, q}\left(i_{q}\right)$ in Querrichtung der untersuchten SynRM (siehe Tab. 1) sind in Abb. 2 gezeigt. Daraus resultieren nach Gleichungen (1) und (2) die Induktivitäten $I_{d}\left(i_{d}\right)$ und $I_{q}\left(i_{q}\right)$, welche in Abb. 3 dargestellt sind. Für sehr

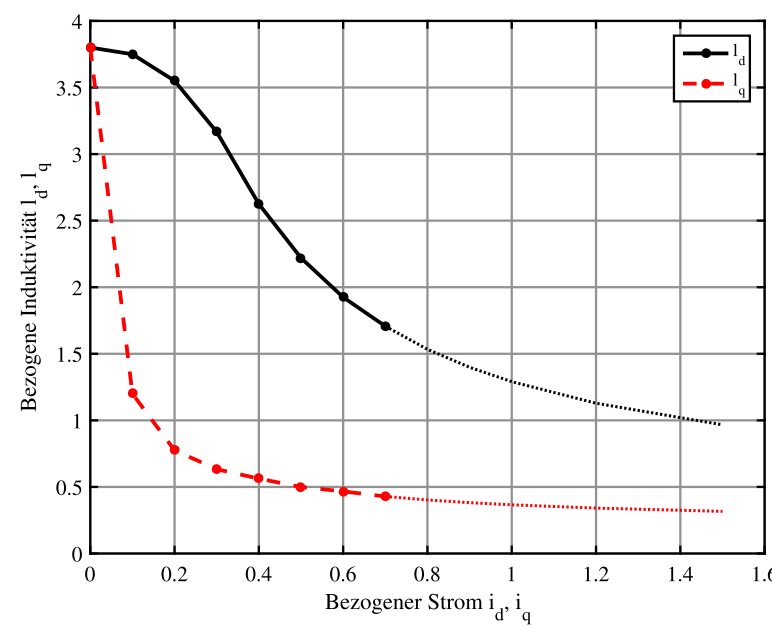

Abb. 3. Induktivitäten $I_{d}$ und $I_{q}$ in Abhängigkeit des jeweiligen Stroms $i_{d}$ bzw. $i_{q}$

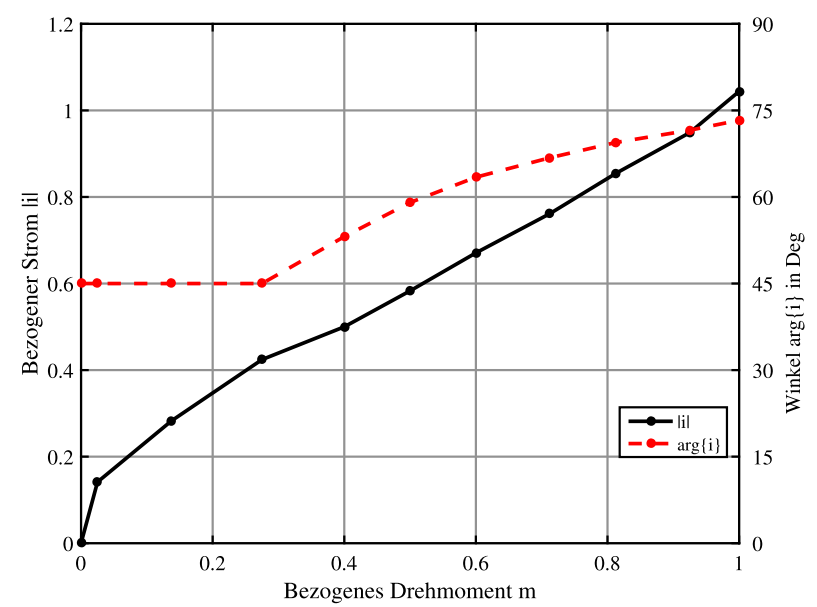

Abb. 4. Strombetrag $\left|i_{s}\right|$ und Stromwinkel $\arg \left\{i_{s, d q}\right\}$

kleine Ströme sind die Induktivitäten gleich, da die Stege im Rotor der Maschine noch nicht gesättigt sind. Bei Erhöhung des Querstroms $i_{q}$ erfolgt rasch eine Sättigung der schmalen Rotorstege wodurch die Induktivität $I_{q}$ sinkt. Aufgrund der unterschiedlichen Induktivitäten erfolgt eine Drehmomentbildung bei gleichzeitiger Bestromung in $d$ - und in $q$-Richtung entsprechend

$$
\begin{aligned}
m & =-\Im m\left\{\underline{\psi}_{S} i_{S}^{*}\right\} \\
& =\psi_{S, d} i_{q}-\psi_{S, q} i_{d} \\
& =\left(I_{d}-I_{q}\right) i_{d} i_{q}
\end{aligned}
$$

Abbildung 4 zeigt die Referenzwerte der Regelung für den Strombetrag $\left|i_{s}\right|$ und den Stromwinkel $\varepsilon=\arg \left\{i_{S, d q}\right\}$. Für kleine Drehmomente ist der Winkel $45^{\circ}$ und steigt für höhere Drehmomente an. Aus der Drehmomentcharakteristik werden die Ströme $i_{d}$ und $i_{q}$ vorgegeben, welche mittels unterlagerter Stromreglung durch den Spannungszwischenkreisumrichter geregelt werden. Für diese Stromregelung ist folglich die Statorspannungsgleichung im rotorfesten $(d, q)$-Koordinatensystem von Interesse.

$$
\underline{u}_{S, d q}=i_{S, d q} r_{S}+\frac{d \underline{\psi}_{S, d q}}{d \tau}+j \omega_{m} \underline{\psi}_{S, d q}
$$


Eine Betrachtung der Spannungskomponenten $u_{d}$ und $u_{q}$ zeigt eine Verkopplung der beiden Gleichungen

$$
\begin{aligned}
& u_{d}=i_{d} r_{s}+I_{d}\left(i_{d}\right) \frac{d i_{d}}{d \tau}-\omega_{m} I_{q}\left(i_{q}\right) i_{q} \\
& u_{q}=i_{q} r_{s}+I_{q}\left(i_{q}\right) \frac{d i_{q}}{d \tau}+\omega_{m} I_{d}\left(i_{d}\right) i_{d}
\end{aligned}
$$

wodurch der Einsatzes eines Entkopplungsnetzwerkes speziell für höhere Drehzahlen im Feldschwächbetrieb empfohlen wird.

\section{Sensorlose Regelung}

In den unterschiedlichen Anwendungsgebieten elektrischer Antriebe bietet die sensorlose bzw. geberlose Regelung Vorteile hinsichtlich Kosten, Baugröße und Fehleranfälligkeit durch die Vermeidung eines Drehzahl- bzw. Positionssensors sowie des dazugehörigen Steckers und der Kabelverbindungen.

SynRM bieten die Möglichkeit, die mit dem Rotor verbundene magnetische Achsigkeit zur Bestimmung des Rotorwinkels heranzuziehen. Die Induktivitätsverhältnisse werden durch Messung der Maschinenströme unter Anregung der Statorspannung ausgewertet. Beispielsweise erfolgt entsprechend [5] die Injektion einer zusätzlichen, grundsätzlich zur feldorientierten Regelung der Maschine nicht benötigten Spannungsharmonischen mit einer bestimmten Trägerfrequenz $f_{C}$. Diese liegt unter Berücksichtigung physikalischer Grenzen in etwa zwischen der doppelten Synchronfrequenz und der Umrichterschaltfrequenz, welche folglich akkustisch hörbar ist. Die gemessene Stromantwort wird entsprechend demoduliert und daraus die Rotorposition ermittelt. Eine Erweiterung dieses sensorlosen Verfahrens zum Erreichen des maximalen Drehmoments durch MTPA-Tracking (Maximum Torque per Ampere) ist in [6] vorgestellt.

Im Gegensatz zur harmonischen Spannungsinjektion mit einer konstanten Frequenz $f_{C}$ werden im klassischen INFORM-Verfahren Spannungspulse in die elektrische Maschine injiziert.

\section{INFORM-Verfahren}

Das sogenannte INFORM-Verfahren (Indirekte Flussermittlung durch Online Reaktanz Messung) kann zur Bestimmung des Rotorlagewinkels von Synchronmaschinen (PSM und SynRM) ohne externen Lagegeber verwendet werden [4]. Eine von der Rotorlage abhängige Induktivität, welche durch Sättigungs- und/oder Reluktanzeffekten hervorgerufen wird, erlaubt die Ermittlung der aktuellen Rotorposition. Anstatt einer periodischen Spannungsinjektion zur Positionsermittlung wird das Pulsmusters des Umrichters zu einer sogenannten 3-aktiven-PWM verändert in der eine Stromanstiegsmessung [7] in allen 3 Strangströmen durchgeführt wird. Damit wird einerseits die Frequenz der Positionsbestimmung erhöht und weiters erfolgt keine akustische Wahrnehmung des sensorlosen Verfahrens, da nun keine zusätzlichen Frequenzen an die Maschine gelegt werden und die Stromantwort aus der PWM-Frequenz ausgewertet wird. Aufgrund der begrenzten Ausgangsspannung der 3-aktiven-PWM wird für höhere Drehzahlen eine symmetrisches Puslmuster verwendet. Die Anwendung sensorloser Verfahren erfordert die Achsigkeit der SynRM. Zur Sicherstellung der Reluktanz ist für diese Maschine entsprechend Abb. 3 eine Mindeststromkomponente von $i_{q}=0.1$ nötig. Die Verwendung eines Rotoraufbaus mit ausgeprägten Polen anstatt Flussbarrieren erlaubt hingegen die geberlose Regelung auch im stromlosen Zustand [8]. Die Positionsermittlung nach dem INFORM-Verfahren verwendet die 3 komplexen Stromänderungen $\Delta \underline{i}_{1}, \Delta \underline{i}_{2}$ und $\Delta \underline{i}_{3}$ zur Ermittlung des komplexen Parameters $\underline{C}_{I N F}$, welcher die Rotorposition $\gamma_{m}$ folgendermaßen repräsentiert.

$$
\underline{C}_{I N F}=\Delta y e^{j 2 \gamma_{m}}
$$

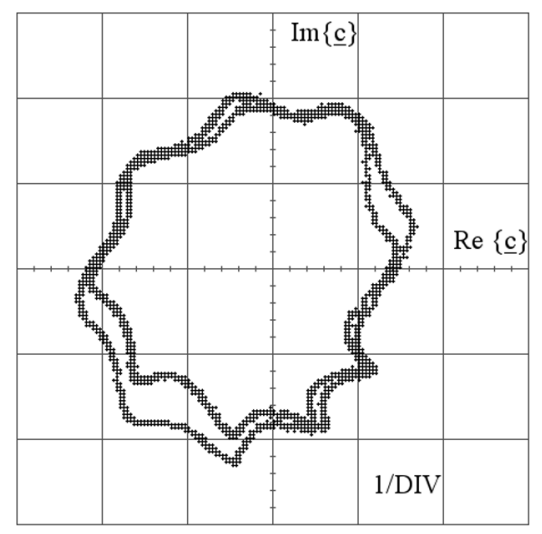

Abb. 5. Messung der INFORM-Ortskurve bei $38 \mathrm{~min}^{-1}$ einer ungeschrägten SynRM, $i_{d}=0.3$ und $i_{q}=1$

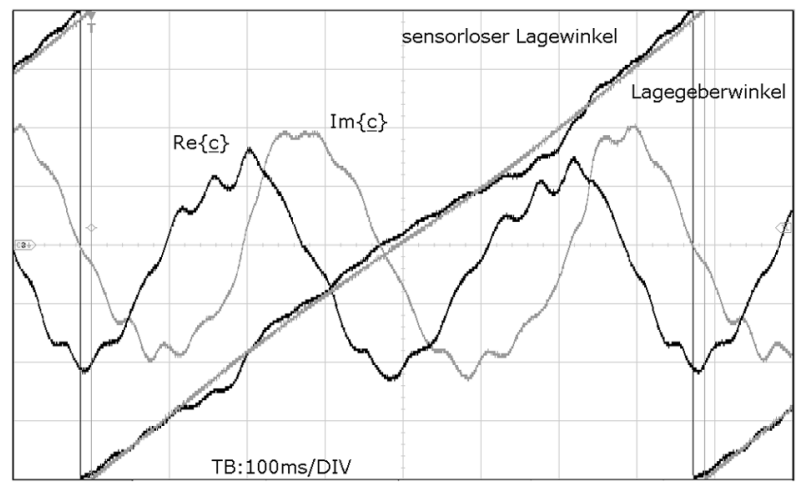

Abb. 6. Zeitverläufe der Real- und Imaginärkomponente von $\underline{\underline{c}}_{I N F}$ sowie daraus ermittelter sensorloser Lagewinkel und Vergleich mit einem Lagegeberwinkel bei Nennmoment

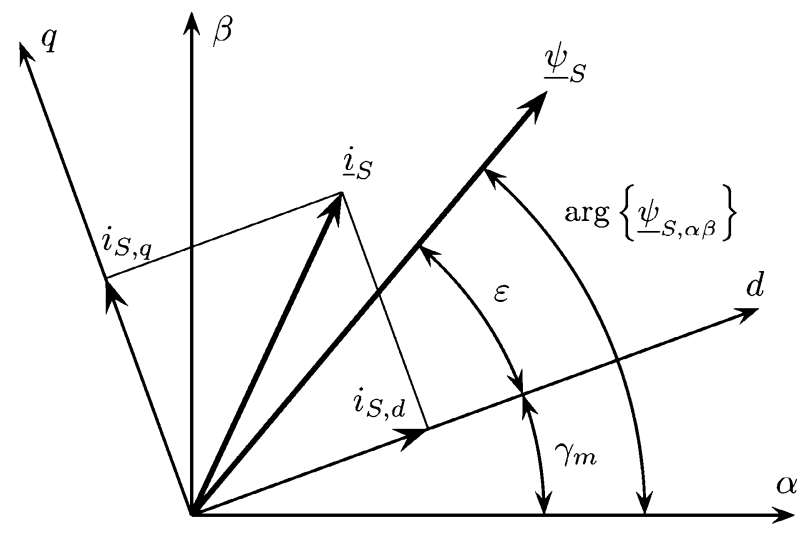

Abb. 7. Raumzeigerdiagramm zur Ermittlung von $\gamma_{m}$ mit dem EMKModell

Die gemessene Ortskurve $\underline{\underline{C}}_{I N F}$ in der komplexen Ebene ist in Abb. 5 dargestellt. Aufgrund des ungeschrägten Maschinenaufbaus sind harmonische Effekte der Stator- bzw. Rotornutung als kleine Ausbeulungen ersichtlich. Eine Messung im sensorlosen Betrieb mit dem INFORM-Verfahren in Abb. 6 zeigt eine geringe Abweichungen zum Lagegeberwinkel. 


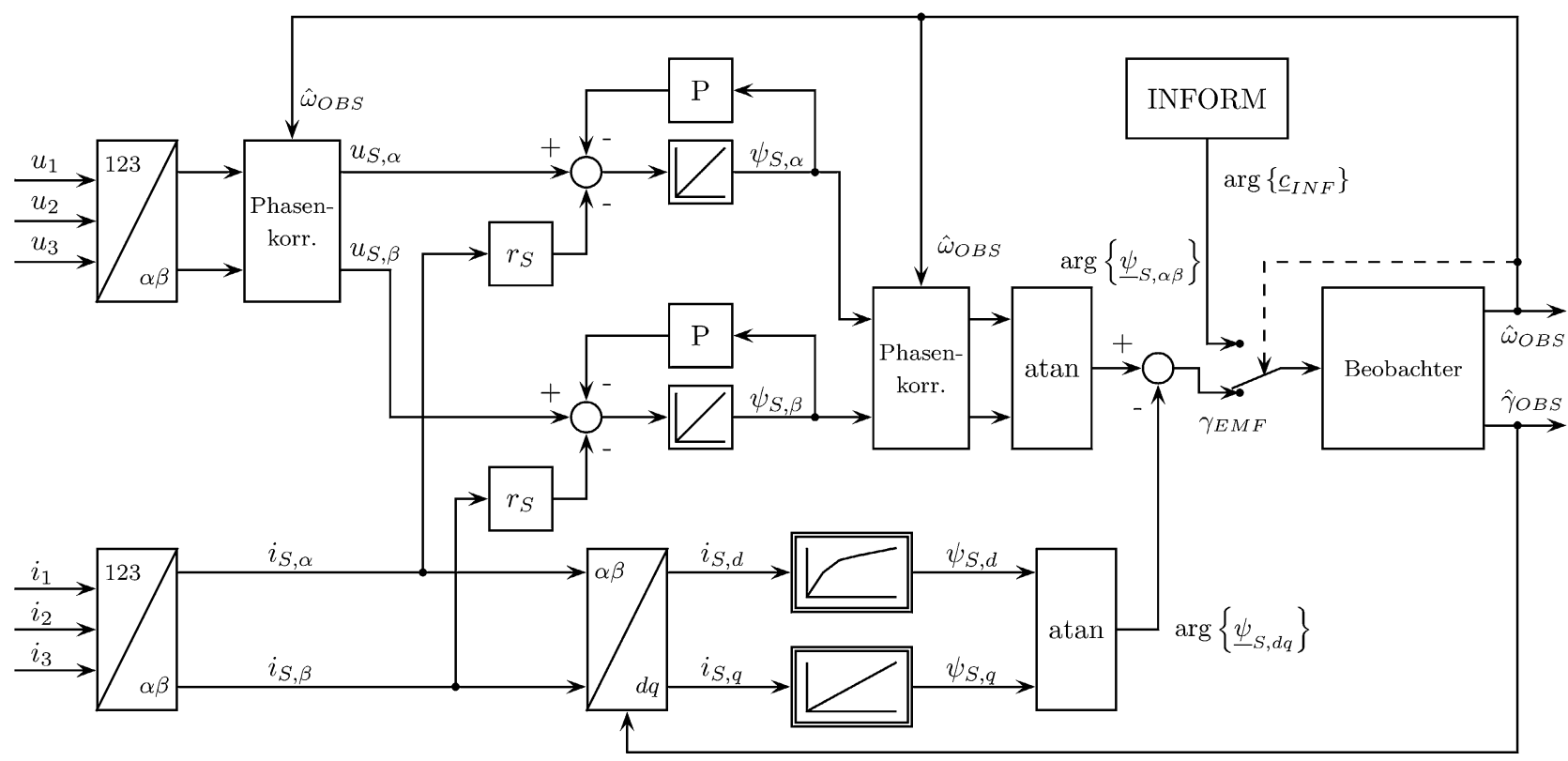

Abb. 8. EMK-Modell für die sensorlose Regelung bei hohen Drehzahlen in Verbindung mit dem INFORM-Verfahren für kleine Drehzahlen und Stillstand

\section{EMK-Modell}

Das EMK-Modell beruht auf der Ermittlung der Rotorposition aus der Elektromotrischen Kraft bei Rotation der Maschine. Die Bestimmung der Statorflussverkettung $\psi_{s}$ erfolgt dabei durch Integration der Statorspannung $\underline{u}_{S}$ abzüglich des ohm'schen Spannungsabfalls am Statorwiderstand $r_{S}$ durch

$$
\frac{d \underline{\psi}_{S}}{d t}=\underline{u}_{S}-r_{S} \underline{i}_{S}
$$

Die Statorspannung $u_{S}$ wird dabei durch eine Spannungsmessung der getakteten Umrichterausgangsspannung in den 3 Phasen $u_{1}$, $u_{2}, u_{3}$ erfasst. Aufgrund eines implementierten Tiefpassfilters ist eine nachfolgende Phasenkorrektur für eine hohe Modellgenauigkeit notwendig. Entsprechend der Abb. 8 erfolgt die Integration mit einer Rückkopplung $P$ für eine Stabilisierung des Modells durch Begrenzung von Driftproblemen. Bei sehr kleinen Drehzahlen bzw. Motorstillstand wird die Lageinformation durch das INFORMVerfahren gewonnen. Der durch die Rückkopplung entstandene Phasenfehler wird mit Hilfe der beobachteten Drehzahl $\omega_{O B S}$ korrigiert. Unter Kenntnis der Flussverkettung $\underline{\psi}_{s, d q}$ wird der Rotorwinkel $\gamma_{m}$ mit dem Winkel $\varepsilon$ nach Abb. 7 folgendermaßen gebildet:

$$
\gamma_{m}=\arg \left\{\underline{\psi}_{S, \alpha \beta}\right\}-\arg \left\{\underline{\psi}_{S, d q}\right\}
$$

Die Flussverkettung $\underline{\psi}_{s, d q}$ erhält man durch Messung des Statorstroms $\underline{i}_{S}$ aus den 3 Strangströmen $i_{1}, i_{2}, i_{3}$ und entsprechender Kenntnis der Magnetisierung in Längsrichtung $\psi_{d}\left(i_{d}\right)$ und Querrichtung $\psi_{q}\left(i_{q}\right)$ unter Berücksichtung des beobachteten Lagewinkels $\gamma_{O B S}$. Ein Beobachter verarbeitet die Winkelinformation aus dem EMK-Modell $\gamma_{E M F}$ oder dem INFORM-Modell $2 \gamma_{\text {INF }}$ und liefert den beobachteten Lagewinkel YOBS und die Drehzahl der Maschine $\omega_{O B S}$, welche zur Bereichsumschaltung zwischen INFORM-Verfahren und EMK- Modell herangezogen wird (Abb. 8). Eine Messung des EMK-Modells nach Abb. 8 bei Nennmoment ist in Abb. 9 dargestellt.

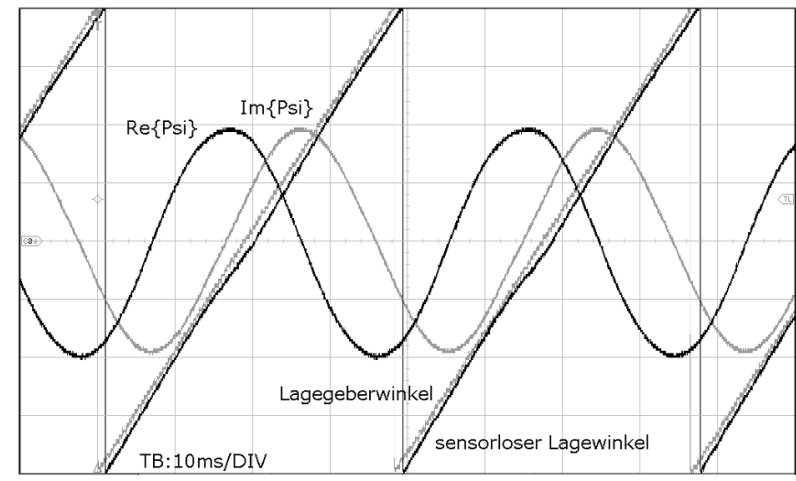

Abb. 9. EMK-Modell: Zeitverläufe der Real- und Imaginärkomponente von $\underline{\psi}_{S, \alpha \beta}$ sowie daraus ermittelter sensorloser Lagewinkel $\gamma_{E M F}$ und Vergleich mit einem Lagegeberwinkel bei Nennmoment

\section{Zusammenfassung}

In dieser Arbeit wurde die sensorlose Regelung einer SynchronReluktanzmaschine unter Verwendung des INFORM-Verfahrens in Kombination mit einem EMK-Modell präsentiert. Damit kann der gesamte Drehzahl- und Lastbereich inklusive Stillstand abgedeckt werden. Der vergleichsweise einfache Maschinenaufbau, kombiniert mit einer geberlosen Regelung, stellt eine kostengünstige Lösung für verschiedene Anwendungen dar. Der sensorlose hochdynamische Betrieb ermöglicht den Ersatz von drehzahlgeregelten Asynchronmaschinen im gesamten Drehzahlbereich einschließlich Stillstand bei höherem Wirkungsgrad und geringerem Systemaufwand aufgrund des Wegfalls eines Lage- bzw. Drehzahlgebers.

Open Access This article is distributed under the terms of the Creative Commons Attribution 4.0 International License (http://creativecommons.org/ licenses/by/4.0/), which permits unrestricted use, distribution, and reproduction in any medium, provided you give appropriate credit to the original author(s) and the source, provide a link to the Creative Commons license, and indicate if changes were made. 


\section{Literatur}

1. Guan, Y., Zhu, Z. Q., Afinowi, I. A. A., Mipo, J. C., Farah, P. (2014): Design of synchronous reluctance and permanent magnet synchronous reluctance machines for electric vehicle application. In The 17th international conference on electrical machines and systems, ICEMS, Hangzhou, China. doi:10.1109/ICEMS.2014.7013785.

2. de Almeida, A. T., Ferreira, F. J. T. E., Baoming, G. (2014): Beyond induction motorstechnology trends to move up efficiency. IEEE Trans. Ind. Appl., 50(3), 2103-2114. doi:10.1109/TIA.2013.2288425.

3. Boglietti, A., Pastorelli, M. (2008): Induction and synchronous reluctance motors comparison. In The 34th annual conference of IEEE industrial electronics, IECON 2008 (S. 2041-2044). doi:10.1109/IECON.2008.4758270.

4. Schrödl, M., Weinmeier, P. (1994): Sensorless control of reluctance machine at arbitrary operation conditions including standstill. IEEE Trans. Power Electron., 9(2), 225-231.

\section{Autoren}

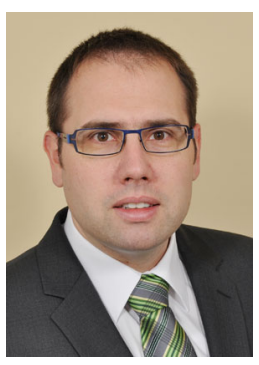

\section{Matthias Hofer}

Studium der Elektrotechnik an der Technischen Universität Wien, Österreich, Studienzweig Automatisierungs- und Regelungstechnik; Abschluss 2004. Universitäts- und Projektassistent am Institut für Elektrische Maschinen und Antriebe der TU Wien 2004 bis 2009, Projektleiter und Abteilungsleiter für die Berechnung elektrischer Maschinen bei Magna Electronics und Magna Powertrain 2009 bis 2013, 2013 Promotion zum Dr. techn., seit 2013 Universitätsassistent am Institut für Energiesysteme und Elektrische Antriebe an der TU Wien.

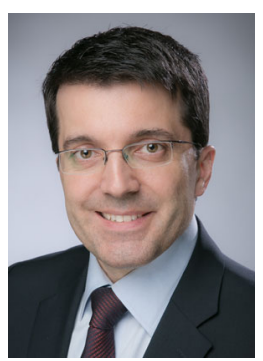

\section{Martin Izaak}

Studium der Elektrotechnik an der Technischen Universität Wien, Österreich, Studienzweig Energie und Antriebstechnik; Abschluss 2004. Universitäts- und Projektassistent am Institut für Elektrische Maschinen und Antriebe der TU Wien 2004 bis 2014, 2009 Promotion zum Dr. techn., seit 2015 V\&V Senior Engineer bei Schneider-Electric Österreich.
5. Villet, W. T., Kamper, M. J., Landsmann, P., Kennel, R. (2012): Evaluation of a simplified high frequency injection position sensorless control method for reluctance synchronous machine drives. In Proceedings of the 6th IET international conference on power electronics, machines and drives, PEMD 2012. ISBN 978-1-84919-616-1.

6. Ide, K., Yamazaki, A. Morimoto, S., lura, H. (2011): Position sensorless control for synchronous reluctance machine based on instantaneous power optimization. In 37th annual conference on IEEE industrial electronics society, IECON 2011 (S. 1977-1982). ISBN 978-1-61284-969-0.

7. Demmelmayr, F., Feischl, M., Troyer, M., Schrödl, M. (2015): Hochdynamische Stromerfassung für elektrische Antriebe mit magnetoresistiven Sensoren. E\&l, Elektrotech. Inf.tech. 132(1), 81-86, doi:10.1007/s00502-014-0276-z.

8. Hofer, M., Spießberger, R., Schrödl, M. (2015): Design and sensorless control of a reluctance synchronous machine for a magnetically levitated drive. In Proceedings of the 2015 IEEE international electric machines and drives conference, IEMDC (S. 916-921). ISBN 978-1-4799-7940-0.

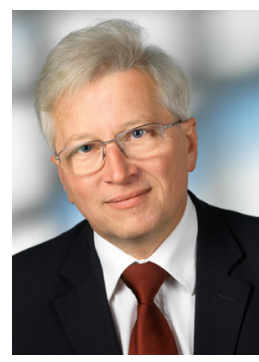

\section{Manfred Schrödl}

Studium der Elektrotechnik an der Technischen Universität Wien, Österreich, Studienzweig Industrielle Elektronik und Regelungstechnik; Abschluss 1982. Universitätsassistent am Institut für Elektrische Maschinen und Antriebe der TU Wien, 1987 Promotion zum Dr. techn., 1992 Habilitation für Elektrische Antriebe und Leistungselektronik in der Antriebstechnik. 1993 bis 1996 Leiter F\&E bei Elin Verkehrstechnik Wien, 1996 bis 1998 Bereichsleiter Zentrale Technik bei Flender ATB, Spielberg, Steiermark. Seit 1998 Ordentlicher Universitätsprofessor für Elektrische Antriebe und Maschinen sowie Vorstand des Instituts für Energiesysteme und Elektrische Antriebe an der TU Wien. 